\section{Into the unknown}

The Invisible Century: Einstein, Freud, and the Search for Hidden Universes

by Richard Panek

Viking: 2004. 288 pp. $\$ 24.99$

\section{Diana Kormos Buchwald}

The title of this book is a little ambiguous: science writer Richard Panek surely meant it to refer to the 'century of the invisible'. Following his earlier account of Galileo's discovery of previously unseen objects in the sky, Seeing and Believing (Penguin, 1998), he now turns to two giants of twentiethcentury scientific and intellectual history, Sigmund Freud and Albert Einstein. He considers how they redefined the way we think about hidden entities and phenomena, in both the deepest recesses of our mind and the farthest reaches of a warped, infinite and expanding Universe.

The telescope, the microscope and other technological advances of the scientific revolution have allowed us to explore both macroscopic and microscopic objects that were previously invisible. Here Panek tells us how Freud and Einstein enabled modern man to rethink the way we think - a claim to an even more profound scientific revolution. Freud's psychoanalysis not only established a method of medical treatment for mild psychological ailments, but sought to understand and explain both the neurological and the psychological structure of the mind. Einstein, by postulating the constancy of the speed of light and the equivalence of the laws of physics in all systems in motion, forced us to rethink, recalculate and eventually abandon some long-entrenched fundamental assumptions about the structure of the Universe, such as the existence of absolute space and time. He led us to understand gravity and mass not merely as newtonian categories but as features inherent in the fabric of his new concept of space-time.

Panek's brief double biography, part history and philosophy of science, part essay on progress and civilization, comes at a time when Einstein is capturing the publishing, popular-science and academic limelight. Exhibitions in the United States, Europe, Israel and elsewhere, as well as conferences, books and articles, have already been devoted to the centennial anniversary next year of Einstein's ground-breaking early papers on special relativity, brownian motion and the photoelectric effect. Some 20 books on Einstein are due to be published in the next year.

Given the complexity of Einstein's scientific work, the general trend of recent Einstein biographies has been to explore his 'human side.' To accomplish this, some authors have resorted to cutting the man down to size — to the scale of folk percep-
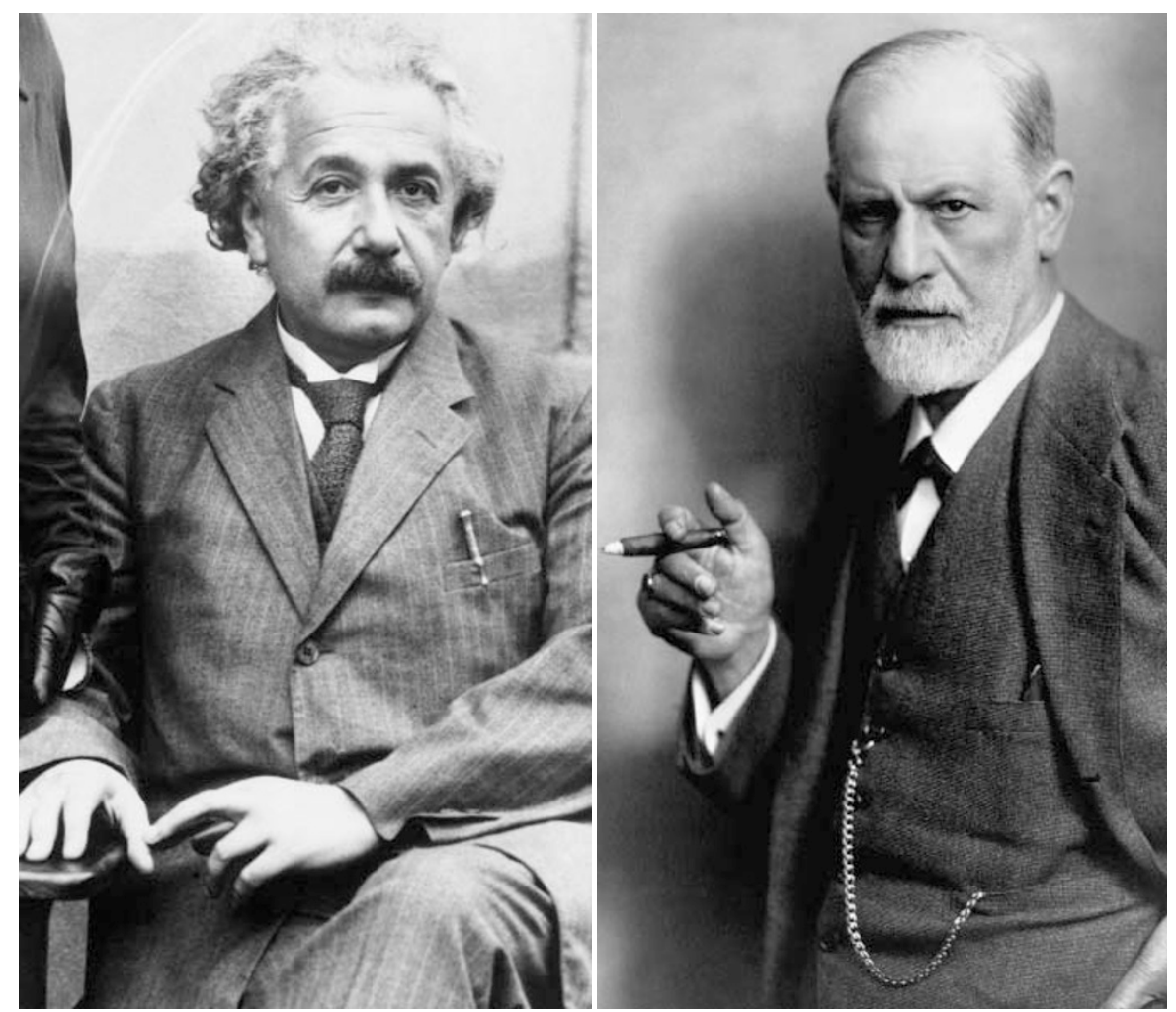

Hidden talent? Albert Einstein (left) and Sigmund Freud both managed to explain the unseen.

tions of morality, goodness, frailty and failure. We have recently learned much about Einstein's illegitimate daughter, his turbulent divorce from his first wife, his extramarital affairs and his inclination not to quote predecessors in scientific papers. A similar treatment was apportioned to Freud in the 1970s. There were many scholarly, as well as journalistic, exposés of Freud's vanity, his competitiveness, his ruthless handling of colleagues, and, most famously, his views on the centrality of fantasy and invention in the sexual-abuse reports of his women patients.

Fortunately, Panek is, if anything, trying to bring some perspective and factual background to the principal achievements of Freud and Einstein, sparing us the moralizing rod. He insists on, and demonstrates, the complexity of reconciling theoretical, mathematical and psychological hypotheses and theories with empirical observations, a profound tenet of both scientists' methodology and creative work.

Both were trained at a time of exhilarating new discoveries in physics, astronomy, medicine and neurology. Both concentrated their efforts on reconceptualizing topics and structures that seemed well explored at the time. And both succeeded in making substantial progress, not by merely seeking answers to unsolved problems, but by asking new questions of old or overlooked material. Why do we routinely misplace keys and umbrellas? What would it be like to travel on a ray of light? Why do people laugh at jokes? How do we know whether we are moving upwards or standing still in an elevator?
What are dreams? What is light? These seemingly trivial fantasies and queries not only allowed the two scientists to escape from the "merely personal", but provided a lever for rearranging long-established experimental and clinical data. Panek covers a great stretch in the history of physics and sciences of the mind, including the concepts of ether and light, X-rays and the mathematization of modern physics. Also there are the discovery of the neuron, the role of hypnosis in Freud's emerging analytic techniques, and his struggle to formulate the analysis of dreams and their relation to the unconscious.

As Panek points out, even though Freud and Einstein corresponded, most notably on the causes of war, and deeply respected each other, they never felt that they were kindred spirits. Freud, in a by now well-known anecdote, reported that, because neither knew much about the other's subject, his meeting with Einstein in 1927 was pleasant enough and free of grandstanding. Panek's book will surely bridge this gap in understanding. It will provide physicists with a cogent and fluid introduction to the early history of psychoanalysis, and to an empathetic reading of Freud and his contemporaries; and its limpid prose will help psychoanalysts, psychologists and modern neuroscientists to understand some of the more complex developments in twentieth-century physics. Both men would have been pleased.

Diana Kormos Buchwald is associate professor of history, and general editor and director of the Einstein Papers Project, California Institute of Technology, Pasadena, California 91125, USA. 